\title{
Impact of Sample Quality to Deep Learning Classification Model of Multiple Crop Types on UAV Remotely Sensed Images
}

\author{
Xingang LI ${ }^{\mathrm{a}, \mathrm{b}}$, Ying CUI ${ }^{\mathrm{a}, \mathrm{b}}$, Xun ZHOU ${ }^{\mathrm{a}, \mathrm{b}}$ and Suhong LIU ${ }^{\mathrm{a}, \mathrm{b}, 1}$ \\ ${ }^{a}$ Faculty of Geographical Science, Beijing Normal University, Beijing 100875, China \\ ${ }^{\mathrm{b}}$ Beijing Key Laboratory of Environmental Remote Sensing and Digital City, Beijing \\ Normal University, Beijing 100875, China
}

\begin{abstract}
Precision agriculture becomes considerable important in agricultural modernization, and thus the demand of accurately extracting crop information from remotely sensed images based unmanned aerial vehicle (UAV) has increased sharply. The most contributing factors of crop classification precision are model selection and samples reliability. Crop recognition models have been obvious optimized under the booming deep learning, while our focus is on the latter factor. The article emphatically explored the best experimental configuration with two verification patterns based on three state-of-the-art image classification models to discuss the impact of sample quality on crop classification accuracy. The patterns referred to different composition ratio of training/verification samples and different spatial resolutions of UAV images. The former experiments revealed that crop types could be better recognized when the number of training sets is greater than or equal to the validation set. By comparing the accuracy different brought by resolutions, the other one illustrated that 40 meters is determined to be the best flight altitude, may balance the recognition accuracy and operating cost.
\end{abstract}

Keywords. Crop classification, convolutional neural network, remote sensing data of UAV

\section{Introduction}

As one of the world's major agricultural countries, China presents a serious contradiction in agricultural resources [1]. The classification of crop type are basic problems in agricultural research that have important research significance and application value [2, $3]$. The demand for accurate and low-cost crop type recognition on farmlands has increased sharply throughout the world $[4,5]$, which exerts major impact on policymaking, food-security and so on [6].

The methods of acquiring crop information mainly include the ground survey method and remote sensing technology [7]. With the development of unmanned aerial vehicles (UAVs), UAV remote sensing technology has been widely used in agricultural

\footnotetext{
${ }^{1}$ Corresponding Author, Suhong LIU, Beijing Normal University, China; E-mail: liush@bnu.edu.cn.

Xingang LI, Ying CUI and Xun ZHOU contribute equally to this paper and should be considered as cofirst author.
} 
remote sensing because of its high data accuracy and short acquisition period [8-12]. Numerous data-driven crop classification models based on UAV images are developed to improve the recognition precision. However, according to large numbers of practices, another factor affecting the accuracy of classification is the data employed in the model training stage. For example, more wavelengths of remote sensing images can help improve accuracy because of their richer ground features [13-18]; more training data also contribute much to a better classification result [19]. In addition to the accuracy, the cost of crop classifying is also vital for practices, but been neglected for a long time. Take UAV-based image classification for example, the higher the altitude, the greater the area covered by a scene, which means the lower cost. However, as the flight altitude increases, the visual features become blurry which confuses the recognition model.

Since almost all public researches related to high-resolution remotely sensed images are obtained under ideal conditions [20-22], few studies focus on the various qualities of samples, which may bring uncertainty to crop recognition model. Therefore, in the article, we discussed the impact of sample quality on crop classification accuracy from two aspects: 1 . the composition ratio of training and verification samples, 2. various spatial resolutions of UAV images samples. To handle the first aspect, we designed experiments with different training and validation sample ratios. To deal with the second aspect, samples with five spatial resolutions $(0.28 \mathrm{~cm}, 0.56 \mathrm{~cm}, 1.12 \mathrm{~cm}, 2.24 \mathrm{~cm}$, and $4.48 \mathrm{~cm})$, correspond to five flight altitudes, and were used in classification tasks. In order to exclude the influence factors of the model, we adopt three commonly used models in our crop classification tasks, which was VGG16, VGG19 and ResNet50.

The results revealed that better precision could be achieved when the number of training sets was greater than or equal to the validation set. The precision decreased sharply when we continued to reduce the number of training samples. The similar phenomenon occurred at altitudes above $40 \mathrm{~m}$, proving the near 40 meters flight altitude was the best choice when balancing recognition accuracy and operating cost.

\section{Data and Methodology}

\subsection{Study Sites and Data}

North China plain, an important production bases of provisions and edible oil in China, with abundant crop types. A typical flat area in North China is select as this crop extraction study experimental area, which locates in Qingyuan County, Baoding City, Hebei Province $\left(115^{\circ} 27^{\prime} 15^{\prime \prime} \mathrm{E}, 38^{\circ} 41^{\prime} 24^{\prime \prime} \mathrm{N}\right)$, with wheat as the main crop and variety vegetable types included.

The study investigates six major types of crop in the mature stage (May 2019, UAV images shown in figure.1), include wheat, cabbage, cauliflower, sunflower, watermelon, and pumpkin (figure.2). Considering the cultivated land is distributes in patches due to the human interference or other individual factors, the type purity is determined by image spatial resolution and object scale $[23,24]$. The appropriate grid unit scale is selected to segment the image into patches. The image patch is used as the experimental dataset to learn and extract the deep features of images. The object categories are obtained by visual interpretation. 
The experimental UAV images are acquired DJI Mavic Pro. The more detailed parameters of UAV and camera are shown in table 1 and 2.

Table 1. UAV parameters

\begin{tabular}{rc}
\hline Parameter & Value \\
\hline Body size & $83 \times 83 \times 198 \mathrm{~mm}($ height $\times$ width $\times$ length $)$ \\
Wheelbase & $335 \mathrm{~mm}$ \\
Body weight & $734 \mathrm{~g}$ \\
Climb speed & $5 \mathrm{~m} / \mathrm{s}$ \\
Cruising speed & $10.0 \mathrm{~m} / \mathrm{s}$ \\
Flight time & $<30 \mathrm{~min}$ \\
Wind resistance & $<12 \mathrm{~m} / \mathrm{s}$ \\
Flight radius & $10000 \mathrm{~m}$ \\
Flight height & $1000 \mathrm{~m}$ \\
Actual height & $10 \mathrm{~m}$ \\
\hline
\end{tabular}

Table 2. Camera parameters

\begin{aligned} & \hline Parameter Value \\ & \hline Sensor $1 / 2.3$ inch CMOS \\ & Effective pixels 12.35 million \\ & Maximum resolution $4000 \times 3000$ pixels \\ & F-number $\mathrm{f} / 2.2 \\ &$ Exposure time $1 / 352 \mathrm{~s} \\ &$ Focal length $5 \mathrm{~mm} \\ &$ Photo size $5 \mathrm{MB} \\ &$\hline\end{aligned}

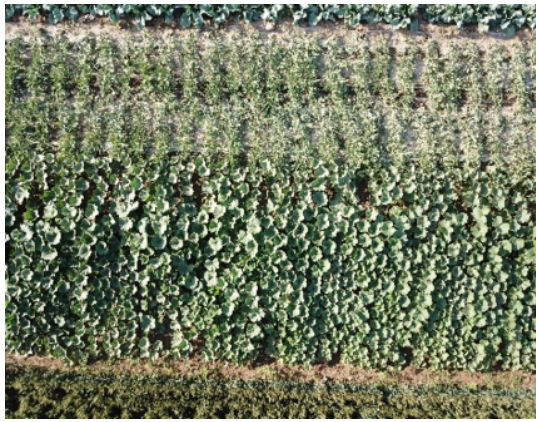

(a)



(c)

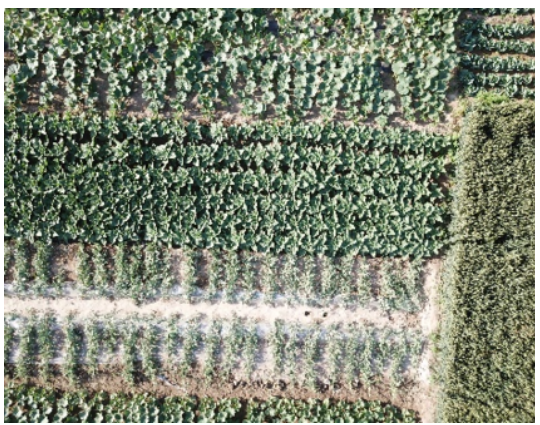

(b)

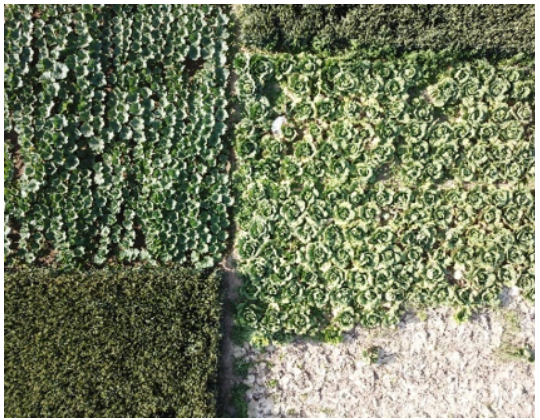

(d) 


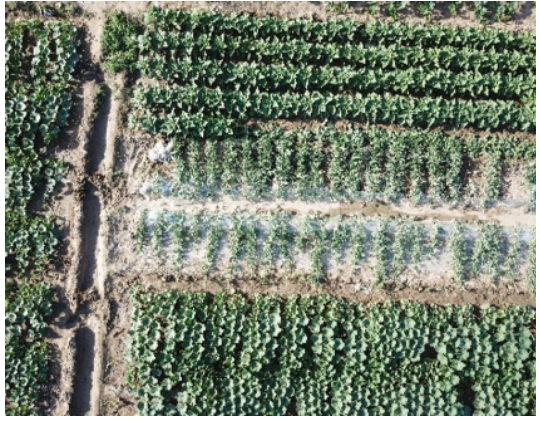

(e)

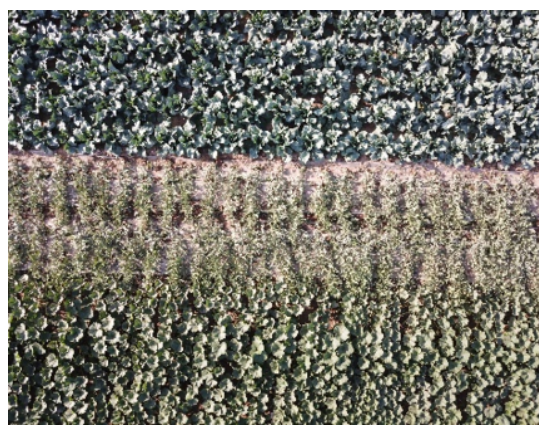

(f)

Figure 1. UAV image data

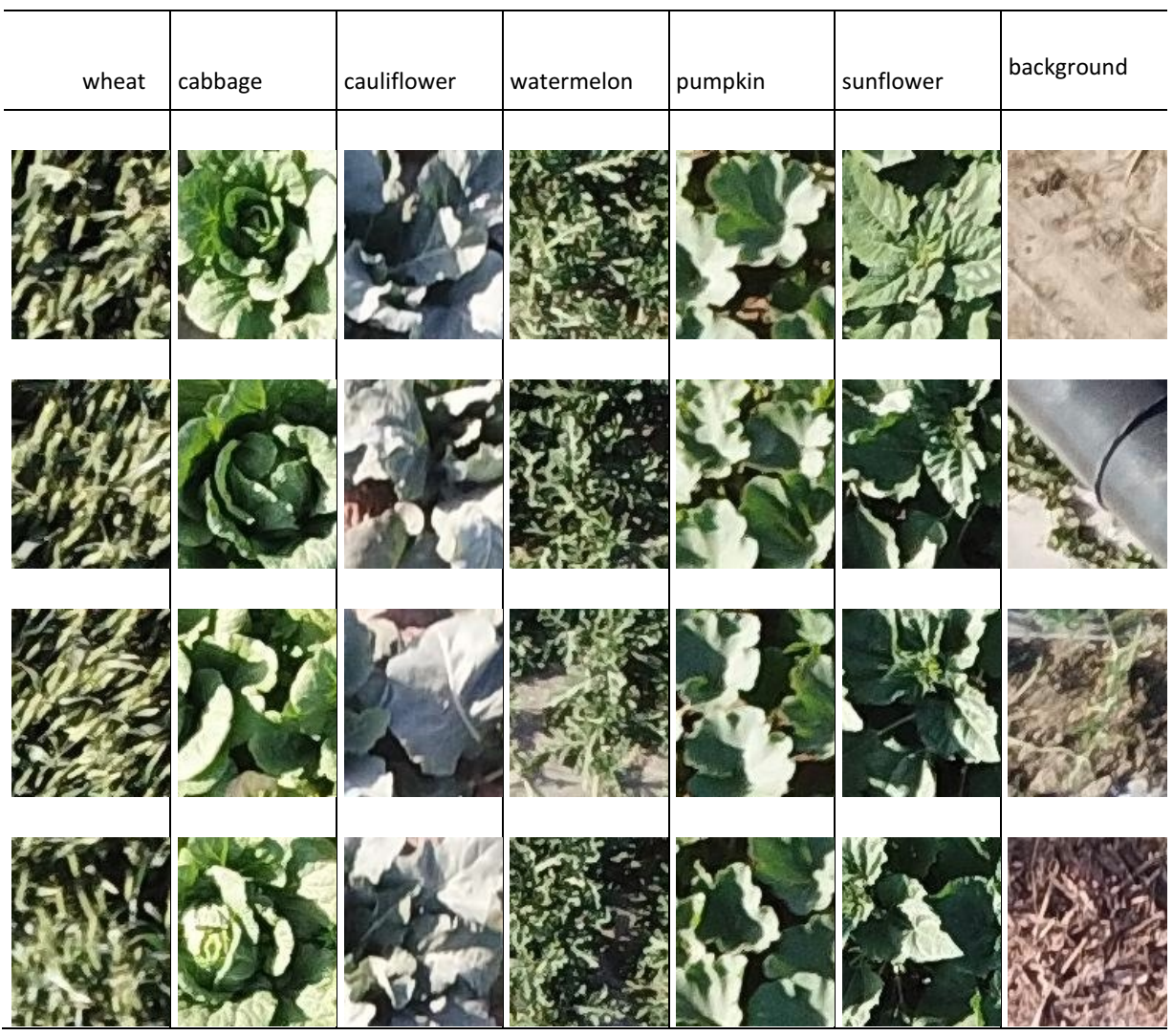

Figure 2. Examples of grid unit types 


\subsection{Methodology}

The method presented in this paper includes the following steps. First, the experimental dataset of the grid unit was constructed by the rule segmentation of the UAV image, which was the training/validation dataset and the test dataset, including the target sample of each type of crop and background sample. Second, the ResNet50, VGG16 and VGG19 models were used to learn the image features of the samples in the training/validation datasets to automatically extract deep features and obtain the optimized network model. Subsequently, the test datasets were classified to obtain the classification results of crop fine recognition and its accuracy evaluation. The reasonable sample size was discussed by changing the training/validation sample ratio, and the reasonable altitude was calculated by resampling different spatial resolution data. The workflow was summarized in figure 3 .
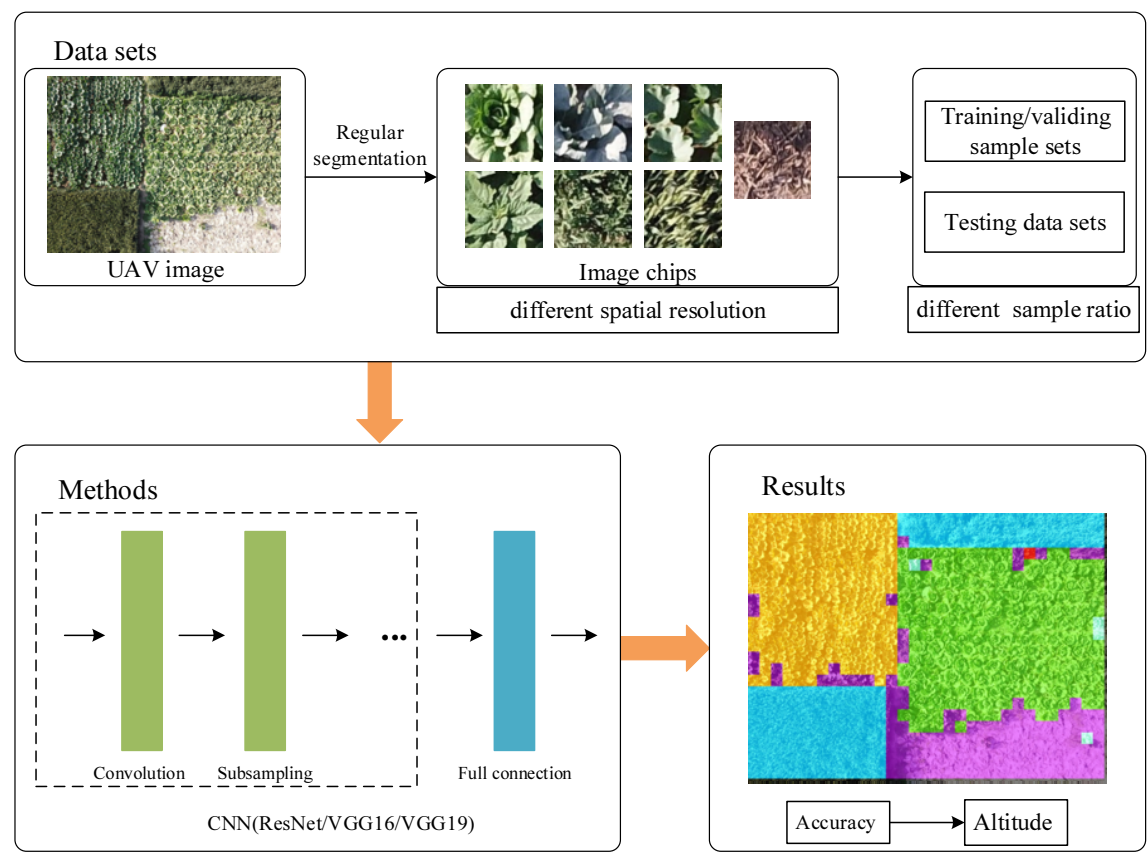

Figure 3. Illustration of the workflow

A Convolution Neural Network is an artificial neural network system with deep learning ability [25]. Compared with traditional image classification, CNNs do not need to extract specific manual features for a specific task but rather simulate the human visual system to handle the original image layer by layer in an abstract manner to generate the classification results [26]. The basic network structure of the CNN can be divided into four parts: input layer, convolutional layer, fully connected layer and output layer [27, 28]. The convolutional layer consists of two parts: convolutional layer and pooling layer (also known as the subsampling layer). The basic structure is shown in figure 4 . 


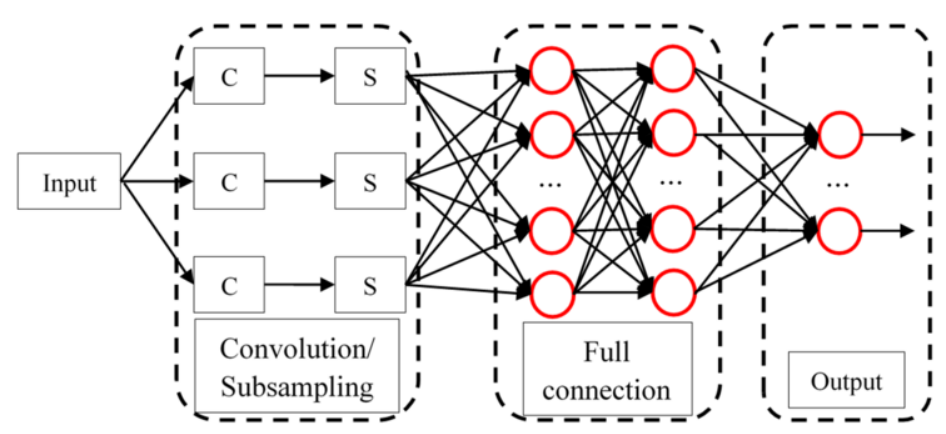

Figure 4. Convolution neural network model

The three models, i.e. VGG16, VGG19 and ResNet50, were used in this study. The main contribution of the VGG-Net is to reveal that the depth of the network is crucial for the performance of the algorithm. VGG16 contains 13 convolutional layers and 3 fully connected layers, while VGG19 contains 16 convolutional layers and 3 fully connected layers. ResNet is a residual learning framework based on the existing training depth network, which can reduce the burden of network training. ResNet50 contains 49 convolutional layers and 1 fully connected layer. The model structures were shown in table 3 .

Table 3. ConvNet configurations of VGG16, VGG19 and ResNet50. The convolutional layer parameters are denoted as "conv(receptive field size)-(number of channels)".

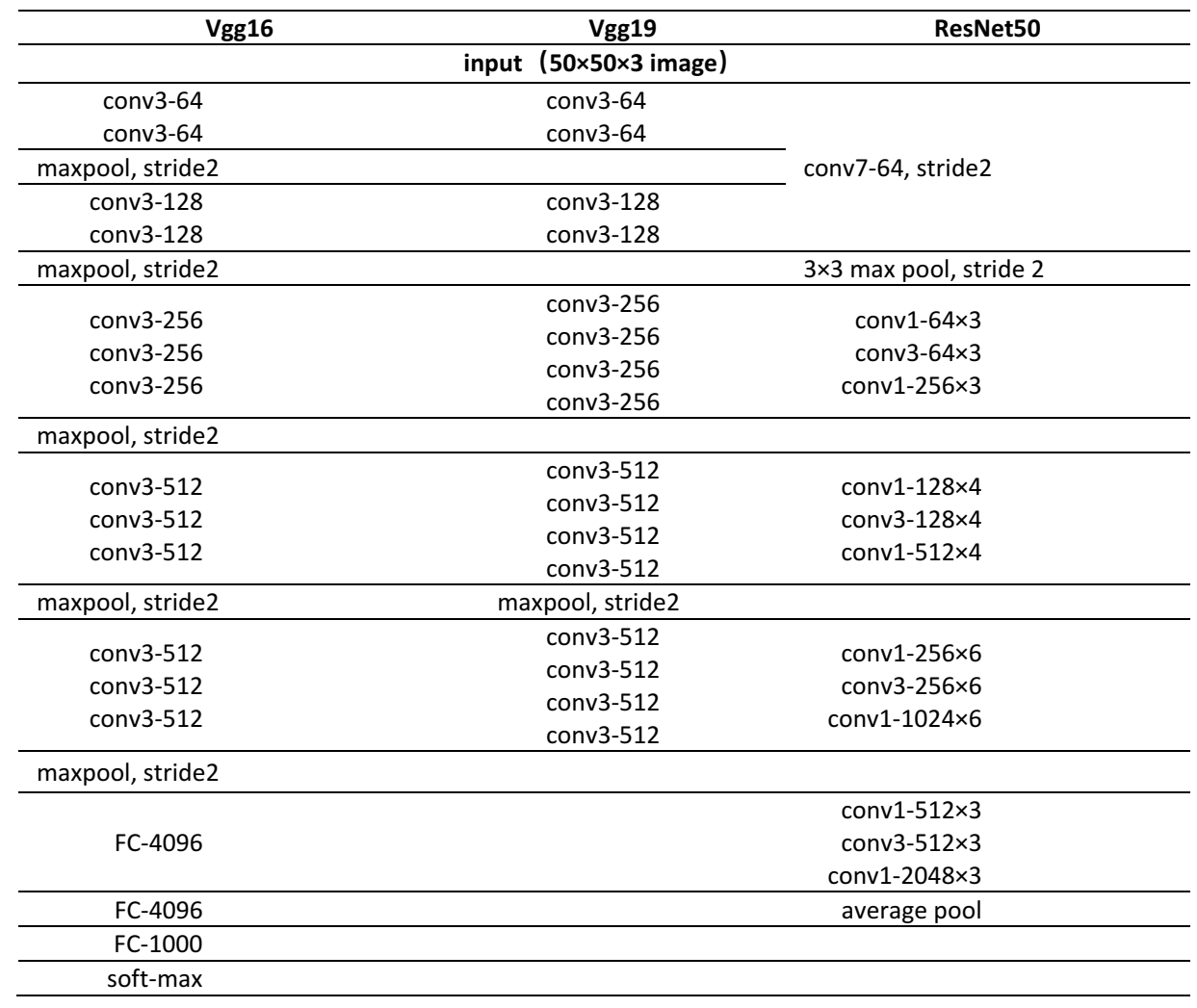


To evaluate the correlation of aerial height and model performance, we built the conversion formula to determine the down-sample rate. The image ground resolution and the aerial height satisfied the following relationship (Eq. (1)):

$$
H=\frac{f \times G S D}{a}
$$

where $\mathrm{H}$ was the aerial height, $\mathrm{f}$ is the lens focal length, GSD was the ground spatial resolution, a was the pixel size and calculated by the size of the sensor's photosensitive element and the resolution of the pixel. According to this equation, the altitude was obtained by resampling simulated data of different spatial resolutions.

\section{Results and Analysis}

\subsection{Experiment}

- Training/validation ratio

A total of 25 scenes of UAV image data were selected, including 22 scenes of training/validation data and 3 scenes of test data. The image patches were acquired by the rule segmentation, and the corresponding training/validation datasets and test datasets were randomly selected. The training data and validation data were distributed proportionally in ratios of $3: 1,2: 2$ and $1: 3$ with different experiments.

- $\quad$ Altitude conditions of aircraft

According to the altitude of the flight experiment $(\mathrm{H}=10 \mathrm{~m})$ and sensor parameters ( $f=5 \mathrm{~mm}, \mathrm{a}=1.39 \mu \mathrm{m}$ ), the ground resolution of the data was $0.28 \mathrm{~cm}$ by Eq. (1). By changing the aggregation scale and resampling the images proportionally, different ground resolution data was simulated. The aggregation scale was $2 \times 2,4 \times 4,8 \times 8$ and $16 \times 16$, and the ground resolution was $0.56 \mathrm{~cm}, 1.12 \mathrm{~cm}, 2.24 \mathrm{~cm}$ and $4.48 \mathrm{~cm}$ respectively, corresponding to the flight altitude of $10 \mathrm{~m}, 20 \mathrm{~m}, 40 \mathrm{~m}, 80,160 \mathrm{~m}$.

\subsection{Result}

The CNN-based VGG16, VGG19 and ResNet50 models were used to classify the training/validation sets. According to the difference of training/validation sample ratios and spatial resolution, the comparative experimental results were obtained. The accuracy of the classification results of test set were evaluated and shown in tables 4, 5, 6 and figure 4 . The three models had good performances in accuracy, precision, recall and $\mathrm{fl-}$ score. In addition, the accuracy of the models changed little with the decrease of the training/validation sample ratio in training stage. Take ResNet-50 model for example, the overall accuracy decreased from 0.97 to 0.96 as the sample ratio changed from $3: 1$ to $1: 3$. However, the accuracy decreased from 0.970 to 0.865 in ResNet-50 model when the image resolution changed from $0.28 \mathrm{~cm}$ to $4.48 \mathrm{~cm}$. The phenomenon also occurred in two other models (0.967 to 0.851 in VGG16 and 0.968 to 0.847 in VGG19). 
Table 4. Classification results of the training/validation datasets based on the ResNet50 model

\begin{tabular}{llccccc}
\hline $\begin{array}{c}\text { Network } \\
\text { model }\end{array}$ & Image resolution & $\begin{array}{c}\text { Training/validation } \\
\text { sample ratio }\end{array}$ & Acc & P(micro) & R(micro) & F1(micro) \\
\cline { 1 - 3 } ResNet-50 & $0.28 \mathrm{~cm}$ & $3: 1$ & 0.9699 & 0.9707 & 0.9756 & 0.9713 \\
ResNet-50 & $0.56 \mathrm{~cm}$ & $3: 1$ & 0.9704 & 0.9732 & 0.9719 & 0.9709 \\
ResNet-50 & $1.12 \mathrm{~cm}$ & $3: 1$ & 0.9584 & 0.959 & 0.9583 & 0.9559 \\
ResNet-50 & $2.24 \mathrm{~cm}$ & $3: 1$ & 0.9411 & 0.9428 & 0.9474 & 0.9405 \\
ResNet-50 & $4.48 \mathrm{~cm}$ & $3: 1$ & 0.8648 & 0.8631 & 0.8602 & 0.8531 \\
\hline ResNet-50 & $0.28 \mathrm{~cm}$ & $2: 2$ & 0.9648 & 0.9669 & 0.9671 & 0.9651 \\
ResNet-50 & $0.56 \mathrm{~cm}$ & $2: 2$ & 0.9639 & 0.9665 & 0.9672 & 0.9644 \\
ResNet-50 & $1.12 \mathrm{~cm}$ & $2: 2$ & 0.9581 & 0.9585 & 0.9619 & 0.9576 \\
ResNet-50 & $2.24 \mathrm{~cm}$ & $2: 2$ & 0.9319 & 0.9325 & 0.9353 & 0.929 \\
ResNet-50 & $4.48 \mathrm{~cm}$ & $2: 2$ & 0.8442 & 0.8422 & 0.8395 & 0.832 \\
\hline ResNet-50 & $0.28 \mathrm{~cm}$ & $1: 3$ & 0.9597 & 0.961 & 0.9636 & 0.9598 \\
ResNet-50 & $0.56 \mathrm{~cm}$ & $1: 3$ & 0.958 & 0.9573 & 0.963 & 0.9571 \\
ResNet-50 & $1.12 \mathrm{~cm}$ & $1: 3$ & 0.9466 & 0.9471 & 0.9486 & 0.9442 \\
ResNet-50 & $2.24 \mathrm{~cm}$ & $1: 3$ & 0.9164 & 0.9201 & 0.9161 & 0.9123 \\
ResNet-50 & $4.48 \mathrm{~cm}$ & $1: 3$ & 0.8148 & 0.8109 & 0.8066 & 0.7962 \\
\hline
\end{tabular}

Table 5. Classification results of the training/validation datasets based on the VGG16 model

\begin{tabular}{lccccccc}
\hline $\begin{array}{c}\text { Network } \\
\text { model }\end{array}$ & Image resolution & $\begin{array}{c}\text { Training/validation } \\
\text { sample ratio }\end{array}$ & Acc & & P(micro) & R(micro) & F1(micro) \\
\cline { 1 - 2 } VGG16 & $0.28 \mathrm{~cm}$ & $3: 1$ & 0.9676 & 0.9696 & 0.9704 & 0.9678 \\
VGG16 & $0.56 \mathrm{~cm}$ & $3: 1$ & 0.9673 & 0.9701 & 0.9691 & 0.9677 \\
VGG16 & $1.12 \mathrm{~cm}$ & $3: 1$ & 0.9571 & 0.9568 & 0.9606 & 0.956 \\
VGG16 & $2.24 \mathrm{~cm}$ & $3: 1$ & 0.9331 & 0.9307 & 0.9395 & 0.9304 \\
VGG16 & $4.48 \mathrm{~cm}$ & $3: 1$ & 0.8513 & 0.8484 & 0.8476 & 0.839 \\
\hline VGG16 & $0.28 \mathrm{~cm}$ & $2: 2$ & 0.9632 & 0.965 & 0.9665 & 0.9635 \\
VGG16 & $0.56 \mathrm{~cm}$ & $2: 2$ & 0.9612 & 0.9635 & 0.9648 & 0.9616 \\
VGG16 & $1.12 \mathrm{~cm}$ & $2: 2$ & 0.9515 & 0.9553 & 0.9542 & 0.9516 \\
VGG16 & $2.24 \mathrm{~cm}$ & $2: 2$ & 0.9233 & 0.9245 & 0.9276 & 0.9216 \\
VGG16 & $4.48 \mathrm{~cm}$ & $2: 2$ & 0.8357 & 0.833 & 0.8363 & 0.824 \\
\hline VGG16 & $0.28 \mathrm{~cm}$ & $1: 3$ & 0.9558 & 0.9549 & 0.9599 & 0.9541 \\
VGG16 & $0.56 \mathrm{~cm}$ & $1: 3$ & 0.9536 & 0.9531 & 0.9576 & 0.9521 \\
VGG16 & $1.12 \mathrm{~cm}$ & $1: 3$ & 0.9448 & 0.9454 & 0.9473 & 0.9427 \\
VGG16 & $2.24 \mathrm{~cm}$ & $1: 3$ & 0.9059 & 0.9091 & 0.9049 & 0.9007 \\
VGG16 & $4.48 \mathrm{~cm}$ & $1: 3$ & 0.80425 & 0.7966 & 0.79652 & 0.7851 \\
\hline
\end{tabular}


Table 6. Classification results of the training/validation datasets based on the VGG16 model

\begin{tabular}{lccccccc}
\hline $\begin{array}{c}\text { Network } \\
\text { model }\end{array}$ & Image resolution & $\begin{array}{c}\text { Training/validation } \\
\text { sample ratio }\end{array}$ & Acc & & P(micro) & R(micro) & F1(micro) \\
\cline { 1 - 2 } VGG19 & $0.28 \mathrm{~cm}$ & $3: 1$ & 0.9681 & 0.9687 & 0.9706 & 0.968 \\
VGG19 & $0.56 \mathrm{~cm}$ & $3: 1$ & 0.9671 & 0.9713 & 0.9704 & 0.969 \\
VGG19 & $1.12 \mathrm{~cm}$ & $3: 1$ & 0.9587 & 0.9615 & 0.9607 & 0.9584 \\
VGG19 & $2.24 \mathrm{~cm}$ & $3: 1$ & 0.9316 & 0.9295 & 0.9362 & 0.9288 \\
VGG19 & $4.48 \mathrm{~cm}$ & $3: 1$ & 0.8469 & 0.8409 & 0.8455 & 0.834 \\
\hline VGG19 & $0.28 \mathrm{~cm}$ & $2: 2$ & 0.9617 & 0.9646 & 0.9642 & 0.9621 \\
VGG19 & $0.56 \mathrm{~cm}$ & $2: 2$ & 0.9604 & 0.9624 & 0.965 & 0.961 \\
VGG19 & $1.12 \mathrm{~cm}$ & $2: 2$ & 0.9518 & 0.9526 & 0.9551 & 0.9503 \\
VGG19 & $2.24 \mathrm{~cm}$ & $2: 2$ & 0.9228 & 0.9224 & 0.9239 & 0.918 \\
VGG19 & $4.48 \mathrm{~cm}$ & $2: 2$ & 0.8304 & 0.8258 & 0.8337 & 0.8192 \\
\hline VGG19 & $0.28 \mathrm{~cm}$ & $1: 3$ & 0.9578 & 0.9579 & 0.9618 & 0.9571 \\
VGG19 & $0.56 \mathrm{~cm}$ & $1: 3$ & 0.9529 & 0.9531 & 0.9591 & 0.9532 \\
VGG19 & $1.12 \mathrm{~cm}$ & $1: 3$ & 0.9406 & 0.9418 & 0.9418 & 0.938 \\
VGG19 & $2.24 \mathrm{~cm}$ & $1: 3$ & 0.9069 & 0.9085 & 0.908 & 0.9018 \\
VGG19 & $4.48 \mathrm{~cm}$ & $1: 3$ & 0.7986 & 0.7955 & 0.7898 & 0.7798 \\
\hline
\end{tabular}

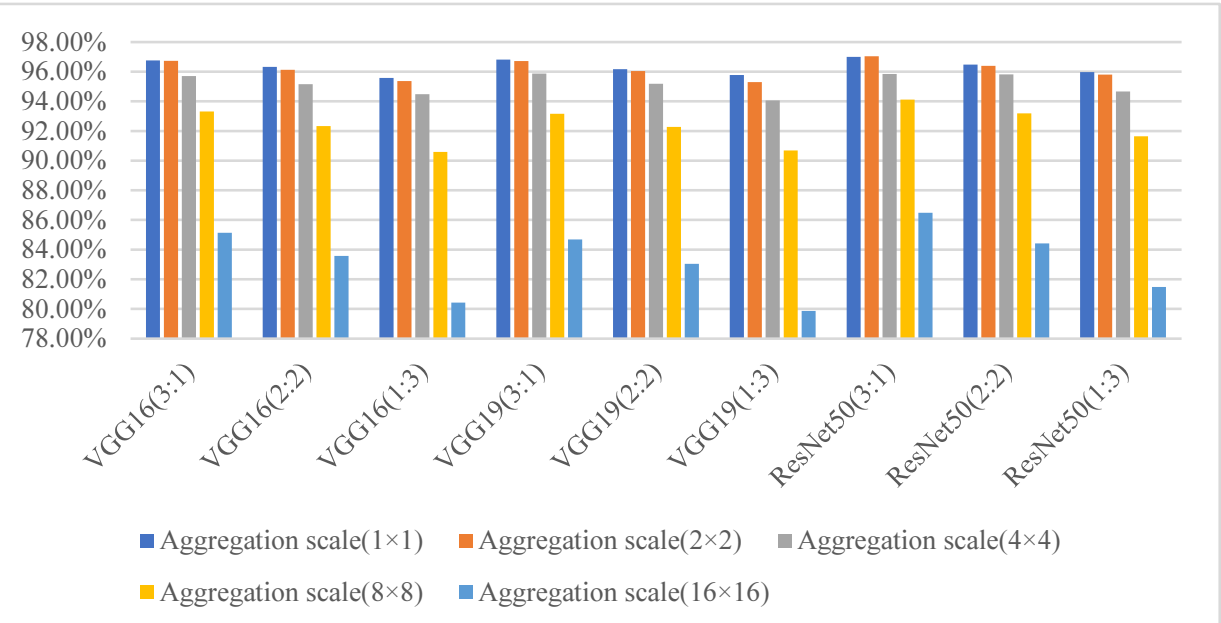

Figure 5. Short caption. Comparisons of the classification results of different training/validation ratio and spatial resolution samples by different models

The test datasets were classified and identified (taking ResNet50 network model as an example) according to the network model of different spatial resolution and training/validation sample ratios. The classification results were shown in figure 5 . The classification results under various conditions were shown in table 7. In figure 6 , the results in test stage were similar to those in training stage. The average accuracy of the three models decreases from 0.922 to 0.893 as the image sampling ratio varies from $3: 1$ to $1: 3$ which was inconsistent with the result in training stage. Particularly, the diversity of image resolution (from $0.28 \mathrm{~cm}$ to $4.48 \mathrm{~cm}$ ) caused $25.9 \%$ reduction in accuracy (from 
0.922 to 0.663 ). We drew the conclusion that the decreases in sampling rates or image resolution magnified the decline in model accuracy when the model was applied to unseen images.

Table 7. Classification results of the training/validation datasets based on the three models

\begin{tabular}{|c|c|c|c|c|c|c|}
\hline Test & $\begin{array}{c}\text { Image } \\
\text { resolution }\end{array}$ & $\begin{array}{l}\text { Training/validati } \\
\text { on sample ratio }\end{array}$ & Acc & $\mathbf{P}$ & $\mathbf{R}$ & F1 \\
\hline \multirow{7}{*}{1} & $0.28 \mathrm{~cm}$ & $3: 1$ & 0.952 & 0.937 & 0.952 & 0.944 \\
\hline & $0.28 \mathrm{~cm}$ & $2: 2$ & 0.949 & 0.936 & 0.949 & 0.942 \\
\hline & $0.28 \mathrm{~cm}$ & $1: 3$ & 0.917 & 0.897 & 0.917 & 0.907 \\
\hline & $0.56 \mathrm{~cm}$ & $3: 1$ & 0.965 & 0.960 & 0.965 & 0.963 \\
\hline & $1.12 \mathrm{~cm}$ & $3: 1$ & 0.960 & 0.960 & 0.960 & 0.960 \\
\hline & $2.24 \mathrm{~cm}$ & $3: 1$ & 0.953 & 0.948 & 0.953 & 0.951 \\
\hline & $4.48 \mathrm{~cm}$ & $3: 1$ & 0.925 & 0.915 & 0.925 & 0.919 \\
\hline \multirow{7}{*}{2} & $0.28 \mathrm{~cm}$ & $3: 1$ & 0.895 & 0.895 & 0.895 & 0.895 \\
\hline & $0.28 \mathrm{~cm}$ & $2: 2$ & 0.886 & 0.886 & 0.886 & 0.886 \\
\hline & $0.28 \mathrm{~cm}$ & $1: 3$ & 0.896 & 0.896 & 0.896 & 0.896 \\
\hline & $0.56 \mathrm{~cm}$ & $3: 1$ & 0.900 & 0.900 & 0.900 & 0.900 \\
\hline & $1.12 \mathrm{~cm}$ & $3: 1$ & 0.881 & 0.881 & 0.881 & 0.881 \\
\hline & $2.24 \mathrm{~cm}$ & $3: 1$ & 0.823 & 0.823 & 0.823 & 0.823 \\
\hline & $4.48 \mathrm{~cm}$ & $3: 1$ & 0.619 & 0.619 & 0.619 & 0.619 \\
\hline \multirow{7}{*}{3} & $0.28 \mathrm{~cm}$ & $3: 1$ & 0.920 & 0.920 & 0.920 & 0.920 \\
\hline & $0.28 \mathrm{~cm}$ & $2: 2$ & 0.909 & 0.909 & 0.909 & 0.909 \\
\hline & $0.28 \mathrm{~cm}$ & $1: 3$ & 0.867 & 0.867 & 0.867 & 0.867 \\
\hline & $0.56 \mathrm{~cm}$ & $3: 1$ & 0.870 & 0.870 & 0.870 & 0.870 \\
\hline & $1.12 \mathrm{~cm}$ & $3: 1$ & 0.741 & 0.741 & 0.741 & 0.741 \\
\hline & $2.24 \mathrm{~cm}$ & $3: 1$ & 0.523 & 0.523 & 0.523 & 0.523 \\
\hline & $4.48 \mathrm{~cm}$ & $3: 1$ & 0.445 & 0.445 & 0.445 & 0.445 \\
\hline \multirow{7}{*}{ Average } & $0.28 \mathrm{~cm}$ & $3: 1$ & 0.922 & 0.917 & 0.922 & 0.920 \\
\hline & $0.28 \mathrm{~cm}$ & $2: 2$ & 0.915 & 0.910 & 0.915 & 0.913 \\
\hline & $0.28 \mathrm{~cm}$ & $1: 3$ & 0.893 & 0.887 & 0.893 & 0.890 \\
\hline & $0.56 \mathrm{~cm}$ & $3: 1$ & 0.912 & 0.910 & 0.912 & 0.911 \\
\hline & $1.12 \mathrm{~cm}$ & $3: 1$ & 0.861 & 0.861 & 0.861 & 0.861 \\
\hline & $2.24 \mathrm{~cm}$ & $3: 1$ & 0.767 & 0.765 & 0.767 & 0.766 \\
\hline & $4.48 \mathrm{~cm}$ & $3: 1$ & 0.663 & 0.659 & 0.663 & 0.661 \\
\hline
\end{tabular}









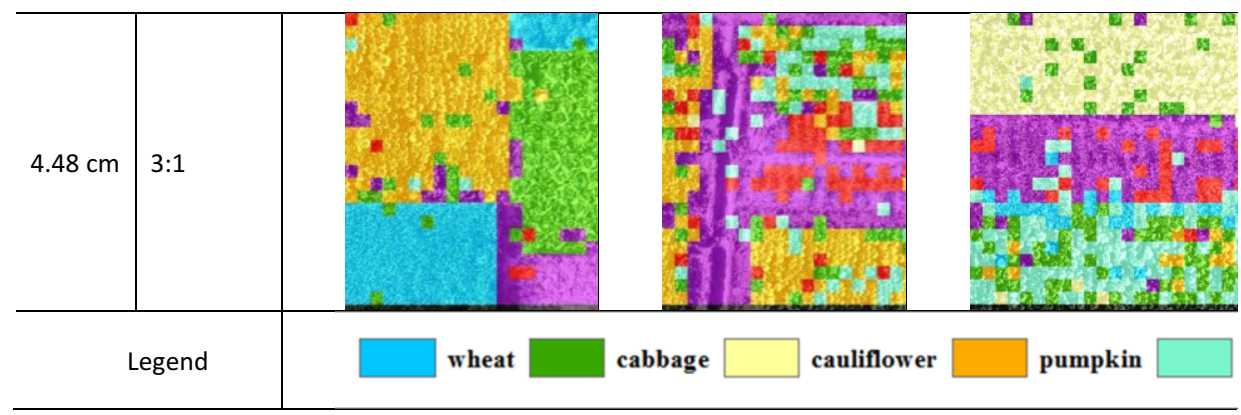

Figure 6. Comparisons of the classification results of test datasets by different models. (Blue pixel represents wheat field; Green pixel represents cabbage field; Light yellow pixel represents cauliflower field; Yellow pixel represents pumpkin field; Light green pixel represents sunflower field; Red pixel represents sweet potato field; Purple pixel represents the background.)

\subsection{Discuss}

- Accuracy comparison under different training/validation ratio

The above tables and figures showed that in the training/validation stage, the classification performance of the CNN-based VGG16, VGG19 and ResNet50 models is better under the conditions of high ground resolution and high training/validation sample ratios. Through further experiments, we found that the decreases in sampling rates or image resolution magnified the decline in model accuracy when the model was applied to unseen images.

- Accuracy comparison under various altitude conditions of aircraft

The result showed that the performance of the classification model is sensitive to the ground resolution of the data. As the flight altitude increases, the classification accuracy of the three models decreases together. When the flight altitude is near $40 \mathrm{~m}$, an obvious inflection point occurs and the classification accuracy decreases sharply. When the aggregation scale is $8 \times 8$ and $16 \times 16$, the classification accuracy of the three models is so low that it cannot meet the actual operation needs. Therefore, the $4 \times 4$ aggregation scale is an obvious threshold, and it can be used to judge whether the minimum ground resolution requirement for fine crop discrimination is $0.28 \times 4=1.12 \mathrm{~cm}$; therefore, that the highest flight altitude is estimated at approximately near $40 \mathrm{~m}$.

\section{Conclusions}

Based on the three commonly used classification model, we discussed the impact of sample quality on crop classification accuracy in UAV visible image data from two aspects: 1. The composition ratio of training and verification samples; 2. Samples with various spatial resolutions. We designed different training and validation sample ratios for the three models and simulated some images by down-sample operation instead of producing them by the aircraft flying at different height. The following conclusions are drawn from the experiments in this paper. 
(1) The CNN-based VGG16, VGG19 and ResNet50 models had ideal classification results for the training/validation datasets with high ground resolution and high training/validation sample ratios, with accuracies up to $97.04 \%$. Thus, the three models are effective for crop type recognition in the study region using UAV visible image data and can provide powerful data support for precision agriculture.

(2) The results in test dataset had more disturbance than that in validation dataset, which indicated that the decreases in sampling rates or image resolution magnified the decline in model accuracy when the model was applied to unseen images.

(3) By changing the aggregation scale to simulate different ground resolution data, the classification performance of the three models decreased sharply as the ground resolution decreases. The models are more sensitive to the ground resolution parameters of the data. The $4 \times 4$ aggregation scale is an obvious threshold, which determined the ground resolution requirement for fine crop discrimination is $0.28 \times 4=1.12 \mathrm{~cm}$. The highest flight altitude is estimated at approximately $40 \mathrm{~m}$.

\section{Acknowledgements}

This research is supported by the National Key Research and Development Project of China (2017YFB0504100).

\section{References}

[1] Lü, X.J.T.o.t.C.S.o.A.E., China's food security and early-warning system based on vector autoregression (VAR) model. 2013. 29(11): p. 286-292.

[2] U1 Qayyum, Z., et al., Optimal Feature Extraction Technique for Crop Classification Using Aerial Imagery, in 2013 International Conference on Information Science and Applications (ICISA). 2013, IEEE.

[3] Zhao, B., et al., A survey on deep learning-based fine-grained object classification and semantic segmentation. International Journal of Automation and Computing, 2017. 14(2): p. 119-135.

[4] Trophic and Guild in Biological Interactions Control, in Progress in Biological Control. 2006, Springer Netherlands.

[5] Abdullahi, H.S., F. Mahieddine, and R.E. Sheriff, Technology Impact on Agricultural Productivity: A Review of Precision Agriculture Using Unmanned Aerial Vehicles, in Wireless and Satellite Systems. 2015, Springer International Publishing. p. 388-400.

[6] Zhiming, F., Y. Ling, and Y. Yanzhao, Temporal and Spatial Distribution Patterns of Grain Crops in the West Liaohe River Basin. Journal of Resources and Ecology, 2014. 5(3): p. 244-252.

[7] Sun, J.J.P.e. and r. sensing, Dynamic monitoring and yield estimation of crops by mainly using the remote sensing technique in China. 2000. 66(5): p. 645-650.

[8] Everaerts, J.J.T.I.A.o.t.P., Remote Sensing and S.I. Sciences, The use of unmanned aerial vehicles (UAVS) for remote sensing and mapping. 2008. 37(2008): p. 1187-1192.

[9] Xiang, H. and L. Tian, Development of a low-cost agricultural remote sensing system based on an autonomous unmanned aerial vehicle (UAV). Biosystems Engineering, 2011. 108(2): p. 174-190.

[10] Watts, A.C., V.G. Ambrosia, and E.A. Hinkley, Unmanned Aircraft Systems in Remote Sensing and Scientific Research: Classification and Considerations of Use. Remote Sensing, 2012. 4(6): p. 1671-1692.

[11] Zhang, C. and J.M. Kovacs, The application of small unmanned aerial systems for precision agriculture: a review. Precision Agriculture, 2012. 13(6): p. 693-712.

[12] Matese, A., et al., Intercomparison of UAV, Aircraft and Satellite Remote Sensing Platforms for Precision Viticulture. Remote Sensing, 2015. 7(3): p. 2971-2990.

[13] Shahin, M.A. and S.J. Symons, Detection of Fusarium damaged kernels in Canada Western Red Spring wheat using visible/near-infrared hyperspectral imaging and principal component analysis. Computers and Electronics in Agriculture, 2011. 75(1): p. 107-112.

[14] Zhang, F., et al., Spectral reflectance properties of major objects in desert oasis: a case study of the Weigan-Kuqa river delta oasis in Xinjiang, China. Environmental Monitoring and Assessment, 2011. 184(8): p. 5105-5119. 
[15] Hunt, E.R., et al., A visible band index for remote sensing leaf chlorophyll content at the canopy scale. International Journal of Applied Earth Observation and Geoinformation, 2013. 21: p. 103-112.

[16] Ozdogan, M., et al., Remote Sensing of Irrigated Agriculture: Opportunities and Challenges. Remote Sensing, 2010. 2(9): p. 2274-2304.

[17] Yan, L. and D.P. Roy, Automated crop field extraction from multi-temporal Web Enabled Landsat Data. Remote Sensing of Environment, 2014. 144: p. 42-64.

[18] Pajares, G., Overview and Current Status of Remote Sensing Applications Based on Unmanned Aerial Vehicles (UAVs). Photogrammetric Engineering \& Remote Sensing, 2015. 81(4): p. 281-330.

[19] Vatsavai, R.R., S. Shekhar, and T.E. Burk. A semi-supervised learning method for remote sensing data mining. in 17th IEEE International Conference on Tools with Artificial Intelligence (ICTAI'05). 2005. IEEE.

[20] Xia, G.-S., et al. DOTA: A large-scale dataset for object detection in aerial images. in Proceedings of the IEEE Conference on Computer Vision and Pattern Recognition. 2018.

[21] Long, Y., et al., Accurate object localization in remote sensing images based on convolutional neural networks. 2017. 55(5): p. 2486-2498.

[22] Li, K., et al., Object detection in optical remote sensing images: A survey and a new benchmark. 2020. 159: p. 296-307.

[23] Chen, L., et al., Evaluation of local features for scene classification using VHR satellite images, in 2011 Joint Urban Remote Sensing Event. 2011, IEEE.

[24] Xia, G.-S., et al., AID: A Benchmark Data Set for Performance Evaluation of Aerial Scene Classification. IEEE Transactions on Geoscience and Remote Sensing, 2017. 55(7): p. 3965-3981.

[25] Krizhevsky, A., I. Sutskever, and G.E. Hinton, ImageNet classification with deep convolutional neural networks. Communications of the ACM, 2017. 60(6): p. 84-90.

[26] Kruthiventi, S.S.S., K. Ayush, and R.V. Babu, DeepFix: A Fully Convolutional Neural Network for Predicting Human Eye Fixations. IEEE Transactions on Image Processing, 2017. 26(9): p. 4446-4456.

[27] Liu, B., et al., A semi-supervised convolutional neural network for hyperspectral image classification. Remote Sensing Letters, 2017. 8(9): p. 839-848.

[28] Qayyum, A., et al., Scene classification for aerial images based on CNN using sparse coding technique. International Journal of Remote Sensing, 2017. 38(8-10): p. 2662-2685. 